\title{
T2K off-axis cross section measurements
}

\section{Raquel CASTILLO FERNÁNDEZ*†}

IFAE, Barcelona

E-mail: rcastillo@ifae.es

The understanding neutrino interaction cross section with nuclei has become a limiting factor for running and future neutrino oscillation experiments. As part of the effort of improving oscillation results, the off-axis near detector of the T2K experiment (ND280) has performed several measurements of muon neutrino cross sections with nuclei at neutrino energies from $500 \mathrm{MeV}$ to few $\mathrm{GeV}$. The most recent results on $\mathrm{T} 2 \mathrm{~K}$ neutrino interaction cross sections measurements are presented as well as current developments and prospects. All the measurements present a good agreement with the predictions of the Monte Carlo neutrino event generators officially that are officially used at $\mathrm{T} 2 \mathrm{~K}$.

16th International Workshop on Neutrino Factories and Future Neutrino Beam Facilities 25 -30 August, 2014

University of Glasgow, United Kingdom

*Speaker.

${ }^{\dagger}$ On behalf of the T2K Collaboration. 


\section{Introduction}

The discovery of the neutrino oscillation phenomenon [1] marked the beginning of a new era for the neutrino physics focused on the precise measurement of the oscillation parameters that appears in the PMNS [2] mixing matrix and the neutrino mass differences. Recently the T2K experiment [3] showed the $v_{e}$ appearance observed with a significance of 7.3 $\sigma$ [4]. This marks the beginning of the search for the $\mathrm{CP}$ violating phase $\left(\delta_{C P}\right)$ in the neutrino sector since $\theta_{13}$ has to be non-zero for the $\mathrm{CP}$ violation to be observable in neutrino oscillation. Determining the value of $\delta_{C P}$ is crucial for an understanding of the neutrino oscillation framework. On the other side, $\delta_{C P}$ could be decisive for solving the matter-antimatter asymmetry through a leptogenesis scenario.

To achieve the required precision on these measurements a better understanding of neutrino beam flux and neutrino cross section is needed. Uncertainty on the neutrino interactions potentially gives large systematical errors for the oscillation parameters. It requires theoretical developments, in particular in understanding the nuclear effects involved in the neutrino-nucleus interaction, and an experimental effort to perform neutrino cross section measurements at different energies and targets. Published data from other experiments show discrepancies with the most common theoretical models, see Figure 1.

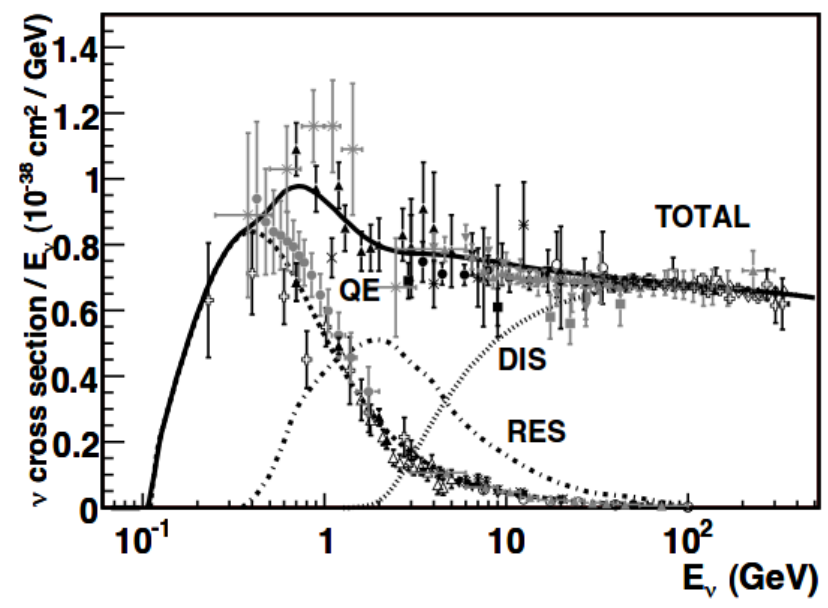

Figure 1: Neutrino cross section values respect to neutrino energy comparing data from several experiments with the predicted distributions from the usual models used, [5].

Neutrino interaction cross sections became important not only to reduce systematical errors on neutrino oscillation studies but for improving our understanding of nuclear theory.

\section{The T2K Experiment}

The Tokai-to-Kamioka (T2K) experiment is a long-baseline neutrino oscillation experiment located in Japan. T2K employs an almost pure muon neutrino $\left(v_{\mu}\right)$ beam. The intense $30 \mathrm{GeV}$ proton beam which is produced at the J-PARC main ring hits the T2K target and produces secondary particles (pions and kaons). The positively charged secondary particles (mainly $\pi^{+}$) are focused 
by three magnetic horns. The $v_{\mu}$ beam is then produced as the decay products of $\pi^{+}$. The beam is measured after oscillation $295 \mathrm{~km}$ away from the production target by the $50 \mathrm{kT}$ water Cherenkov detector Super-KamiokaNDE (SK). The neutrino beam is detected before oscillating by a first set of detectors located $280 \mathrm{~m}$ from the production target called INGRID and ND280.

The SK and ND280 detectors are installed in an off-axis configuration, 2.5 degrees away from the beam center. This off-axis configuration enhances the neutrino oscillation probability by narrowing the neutrino energy distribution around $600 \mathrm{MeV}$ at the oscillation maximum. An overview of the T2K experiment is shown in Figure 2. The resulting neutrino energy spectrum at SK has a narrow-band and it peaks at $0.6 \mathrm{GeV}$.

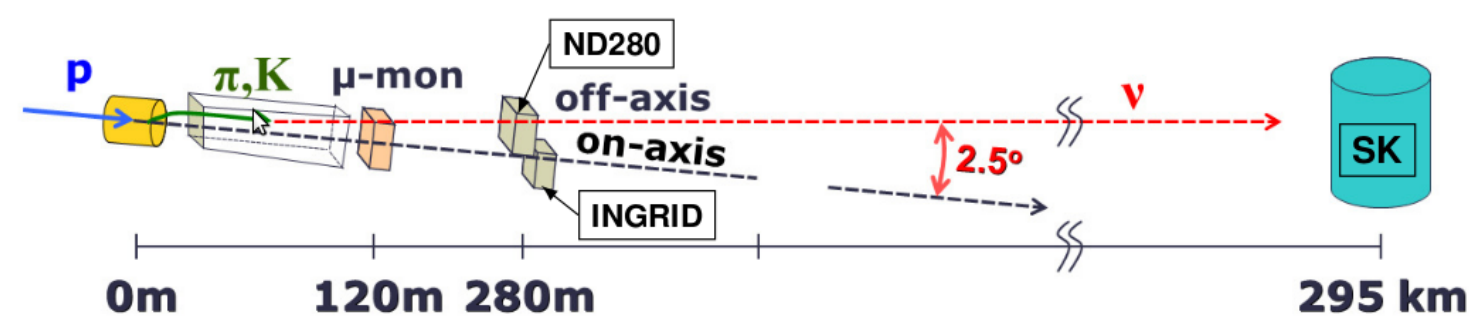

Figure 2: Overview of the T2K experiment.

\section{The off-axis near detector, ND280}

ND280, Figure 3 left, uses a magnet which was previously used for UA1. The magnet produces a magnetic field of $0.2 \mathrm{~T}$. The inner part of ND280 is divided into a tracker section and a $\pi^{0}$ detector. The tracker section are composed of two FGDs (Fine Grained Detectors [6]) and three TPCs (Time Projection Chamber [7]). The most upstream FGD (FGD1) consists of layers of plastic scintillator bars. FGD1 provides the target mass and reconstruction of the short-ranged tracks near the vertexes. FGD2 is divided into scintillator and water modules. The TPC use a mixture gas of Ar:CF4 :iC4 H10 (95:3:2 by mass). They provide particle identification (PID) based on the $d E / d x$ analysis and momentum of the charged particle from the track curvature by the magnetic field. The $\pi^{0}$ detector called P0D [8] consists of plastic scintillators, brass sheets and water bags. The detector is installed to measure the neutrino interaction producing $\pi^{0}$ by using the water target.

The neutrino energy spectrum at ND280 is similar with that at SK. INGRID and the Proton Module are located at on-axis (beam-axis). The neutrino energy spectrum at INGRID (and the Proton Module) has a wide-band compared to that at ND280 and it peaks at $1 \mathrm{GeV}$. The flux prediction for ND280 is showed in Figure 3 right.

\section{Cross section measurements at the ND280 near detector}

With the ND280 complex some cross section measurements at the intermediate energy region from $\sim 500 \mathrm{MeV}$ to a few $\mathrm{GeV}$ have been performed and more ND280 new results are arriving soon. 

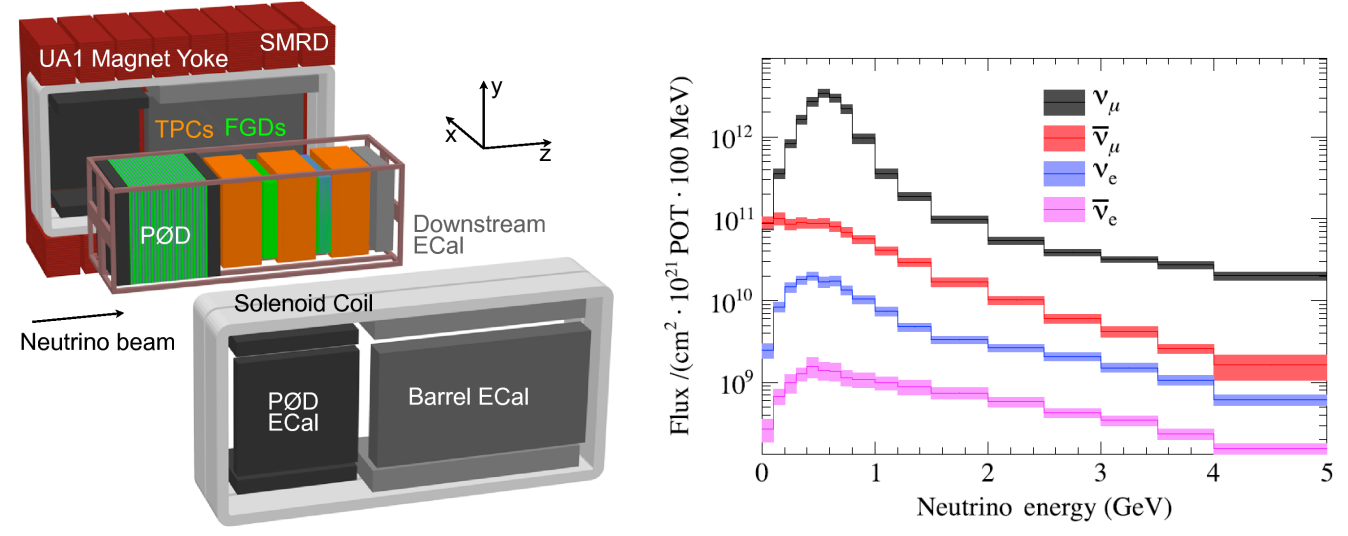

Figure 3: Schematic view of the ND280 detector in left figure. Here the Magnet is open. In right figure, the ND280 flux prediction with systematic error bars, for each neutrino species, is shown. The prediction takes into account the correct relative fractions of 2010 and 20111 beam conditions.

ND280 is used to constrain uncertainties at SK for flux and cross section parameters, jointly with external data. A topological description is used for the event categorization based upon the number of particles leaving the nucleus. Three exclusive samples, Figure 4, are defined according the number of pions in the Final State of the Interaction (FSI).

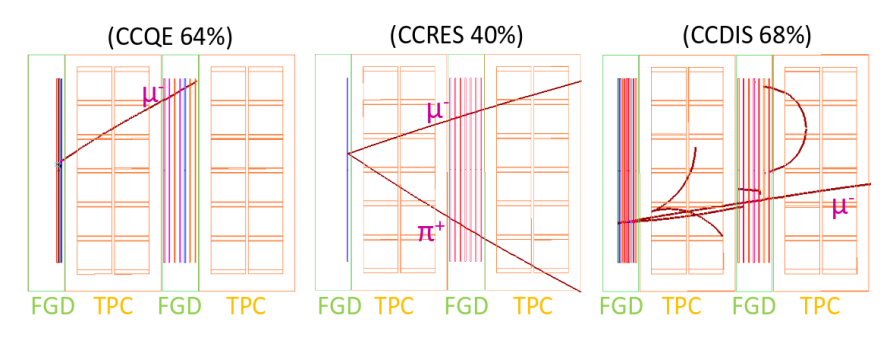

Figure 4: Charged current exclusive samples are separated by topology type used in the official ND280 analysis for oscillation studies joint with SK data. Here, main component of each sub-sample is showed: CCQE (which is the main component of CC0 $\pi$ sub-sample), CC-RES (which is the main component of $\mathrm{CC} \pi^{+}$sub-sample) and CC-DIS (which is the main component of CCOthers sub-sample).

\subsection{Inclusive $v_{\mu}$ Charged Current Cross Section Measurement on Carbon}

The $v_{\mu}$ charged current (CC) inclusive cross section on $\mathrm{CH}$ is measured using the tracker section in the ND280. The interactions are selected by requiring events with a reconstructed $\mu^{-}$ starting in the FGD1 fiducial volume. This selection has a purity of $87 \%$ of CC inclusive signal and an efficiency of $\sim 50 \%$. The flux averaged cross section is extracted by using a Bayesian unfolding method and performing background subtraction based upon the Monte Carlo background prediction (NEUT is the Monte Carlo generator used). The total protons on target (PoT) used in this analysis is $1.08 \times 10^{20}$ and the result for the total flux averaged CC inclusive cross section is: 


$$
\left.\left\langle\sigma_{C C}\right\rangle=6.91 \pm 0.13 \text { (stat. }\right) \pm 0.84(\text { syst. }) \times 10^{-39} \mathrm{~cm}^{2} / \text { nucleon }
$$

for a mean neutrino energy of $0.85 \mathrm{GeV}$ [9]. This result is consistent with theoretical predictions:

$\left\langle\sigma_{N E U T}\right\rangle=7.27 \times 10^{-39} \mathrm{~cm}^{2} /$ nucleon $\left\langle\sigma_{G E N I E}\right\rangle=6.54 \times 10^{-39} \mathrm{~cm}^{2} /$ nucleon

Figure 5 shows the flux averaged cross section result. Results were provided also also in the double differential form $\frac{d \sigma}{d \operatorname{Cos} \theta_{\mu} d p_{\mu}}$.

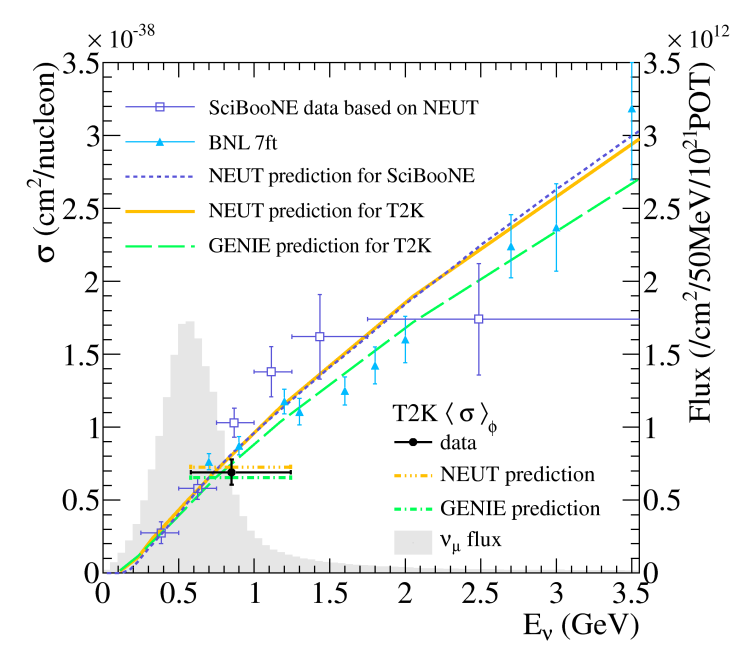

Figure 5: The T2K $v_{\mu} \mathrm{CC}$ inclusive cross section on $\mathrm{CH}$ with NEUT and GENIE prediction and the external experiment. The T2K flux at ND280 is shown in grey.

\subsection{Inclusive $v_{e}$ Charged Current Cross Section Measurement on Carbon}

This is the first $v_{e} \mathrm{CC}$ inclusive cross section measurement at the $\mathrm{GeV}$ scale since the Gargamelle experiment in 1978. The $v_{\mu}$ vs the $v_{e}$ interaction rates are crucial for sensitivity studies. The analysis is performed using the tracker section in ND280. The flux averaged cross section is extracted by using a Bayesian unfolding method as it was done for the $v_{\mu}$ analysis. The total proton on target (PoT) used in this analysis is $5.9 \times 10^{20}$. The result has been provided in the different differential forms: $\frac{d \sigma}{d p_{e}}, \frac{d \sigma}{d \operatorname{Cos} \theta_{e}}, \frac{d \sigma}{d Q^{2}}$. For the last variable, $Q^{2}$, the charged current quasi-elastic (CCQE) interaction is assumed and a phase-space reduction is applied considering efficiencies in angle and electron momentum [10].

Figure 6 shows the flux averaged cross section result and on Figure 7 two of the differential results are shown.

\subsection{Exclusive $v_{\mu}$ Charged Current Quasi-Elastic Cross Section Measurement on Carbon}

The charged current quasi-elastic (CCQE) scattering is the dominant interaction in the T2K experiment. This mode is a two-body interaction:

$v_{\mu}+n \rightarrow \mu+p$

For this interaction channel the neutrino energy in the initial state can be estimated by measuring the momentum and scattering angle of the muon. The CCQE cross section on $\mathrm{CH}$ is measured 


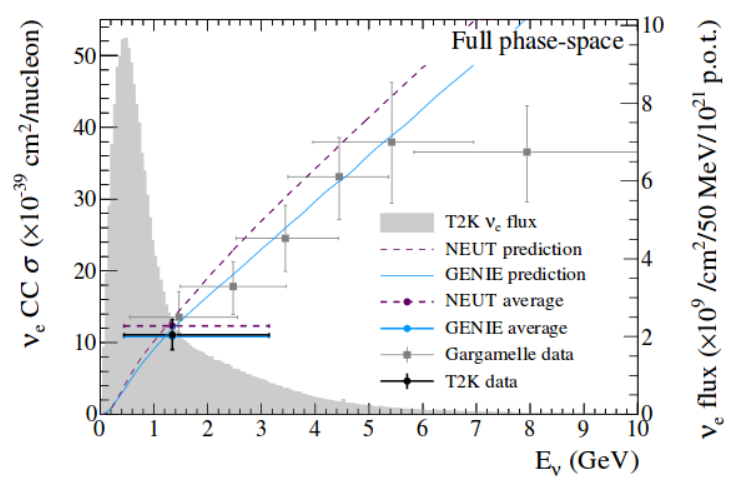

Figure 6: The T2K $v_{e} \mathrm{CC}$ inclusive cross section on $\mathrm{CH}$ with NEUT and GENIE prediction and the external experiment. The T2K flux at ND280 is shown in grey.
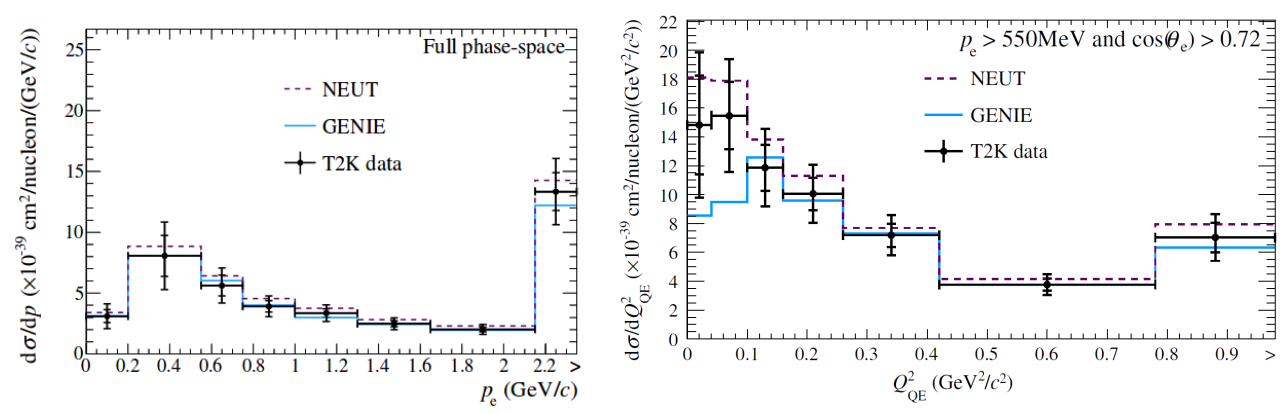

Figure 7: The T2K $v_{e} \mathrm{CC}$ inclusive differential cross section on $\mathrm{CH}$ with NEUT and GENIE prediction. Left plot shows the $\frac{d \sigma}{d p_{e}}$ result, right plot shows the $\frac{d \sigma}{d Q^{2}}$ result where the phase space have been applied.

at the tracker section. The CCQE events are selected by applying an additional cut to the CC inclusive sample in order to select the interaction containing a muon-like and no pions in the final state. The efficiency and purity for the selection is estimated to be $40 \%$ and $72 \%$, respectively. The result [11] and prediction from the NEUT model with a total of $2.63 \times 10^{20}$ PoT is shown in Figure 8. A $\chi^{2}$ test comparing the fitted result with the model is performed and it gives p-value of $17 \%$. The result is consistent with the model.

Currently some new T2K analysis are ongoing for the study of the $C C 0 \pi$ topology: a CCQE double differential measurement with improved event reconstruction and several studies on the 2p2h (two-particle two-holes, that are multi-nucleon effects) effect which is included in the new NEUT MC release.

\subsection{Exclusive $v_{\mu}$ Charged Current Single Pion Cross Section Measurement on Carbon}

As part of the $\mathrm{T} 2 \mathrm{~K}$ effort on neutrino cross sections there is ongoing a $v_{\mu}$ exclusive $\mathrm{CC}$ single pion $\left(\mathrm{CC} \pi^{+}\right)$cross section measurement on $\mathrm{CH}$ using the tracker section in ND280. The interactions are selected by requiring events with a reconstructed $\mu^{-}$, a $\pi^{+}$, no electrons/positrons or pions and any number of nucleons. Tracks start in the FGD1 fiducial volume. the muon is reconstructed 


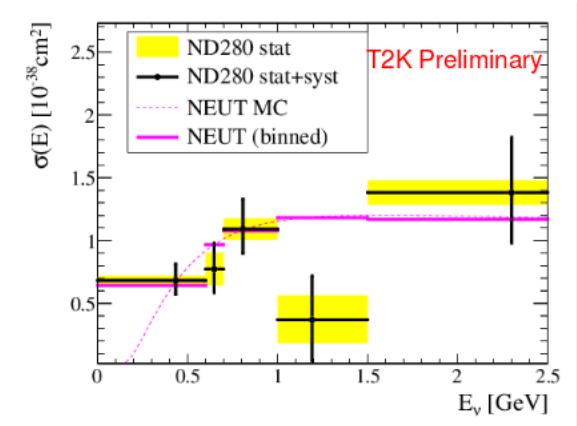

Figure 8: The T2K CCQE cross section on $\mathrm{CH}$ with NEUT prediction.

using FGD1-TPC2, the pion is reconstructed in FGD1-TPC2 or as a delayed cluster in FGD1. This selection has a purity of $\sim 60 \%$. The flux averaged cross section is extracted by using a Bayesian unfolding method and three control samples are used to fit and subtract the background in order to make a more model independent measurement. Result will be provided with respect to several variables: $\frac{d \sigma}{d \operatorname{Cos} \theta_{\mu} d p_{\mu}}, \frac{d \sigma}{d p_{\mu}}, \frac{d \sigma}{d p_{\pi}}, \frac{d \sigma}{d \theta_{\pi}}, \frac{d \sigma}{d Q^{2}}, \frac{d \sigma}{d \mid Q_{3}}, \frac{d \sigma}{d \theta_{\mu \pi}}$, and more angular distributions as well as for $\sigma(E)$.

This measurement is now close to being finalized and it will be provided with an analogous $C C \pi^{+}$measurement on water using FGD2 water modules as the target. The FGD2 analysis uses same signal definition and technique.

On Figure 9 the double differential $\frac{d \sigma}{d \operatorname{Cos} \theta_{\mu} d p_{\mu}}$, for the FGD1 analysis (on CH) is shown when using NEUT to produce fake data and unfolded with NEUT.

\section{5 $v_{\mu}$ Neutral Current Elastic Cross Section Measurement per Nucleon}

The neutral current elastic (NCE) cross section $\left(v_{\mu}+p \rightarrow v_{\mu}+p\right)$ on water is measured with the POD. In order to select proton tracks, the PID based on $d E / d x$ analysis is applied to the events occurring at the POD fiducial volume. The efficiency of $13.7 \%$ and purity of $45.6 \%$ are achieved for this selection. The flux averaged NCE cross section with a total of $9.92 \times 10^{19}$ PoT is measured to be $\left\langle\sigma_{N C E}\right\rangle=2.24 \pm 0.07(\text { stat })_{-0.63}^{+0.53}$ (syst. $) \times 10^{39} \mathrm{~cm}^{2} /$ nucleon [12]. It is consistent with the predictions from NEUT, $\left\langle\sigma_{N C E}\right\rangle=2.02 \times 10^{39} \mathrm{~cm}^{2} /$ nucleon, and GENIE, $\left\langle\sigma_{N C E}\right\rangle=$ $1.78 \times 10^{39} \mathrm{~cm}^{2} /$ nucleon.

\section{Conclusions}

The T2K experiment has performed the neutrino cross section measurements at the off-axis near detector with different targets for the main interaction channels at the T2K energies. All the results are in agreement with the predictions of the neutrino models. Several new analysis are currently ongoing for for $\mathrm{CC} \pi^{+}$cross section on carbon and water and improved measurements for the CCQE. There have been improvements on NEUT which we expect they will be useful for the precise measurements of the neutrino oscillation parameters, and therefore, $\delta_{C P}$. 

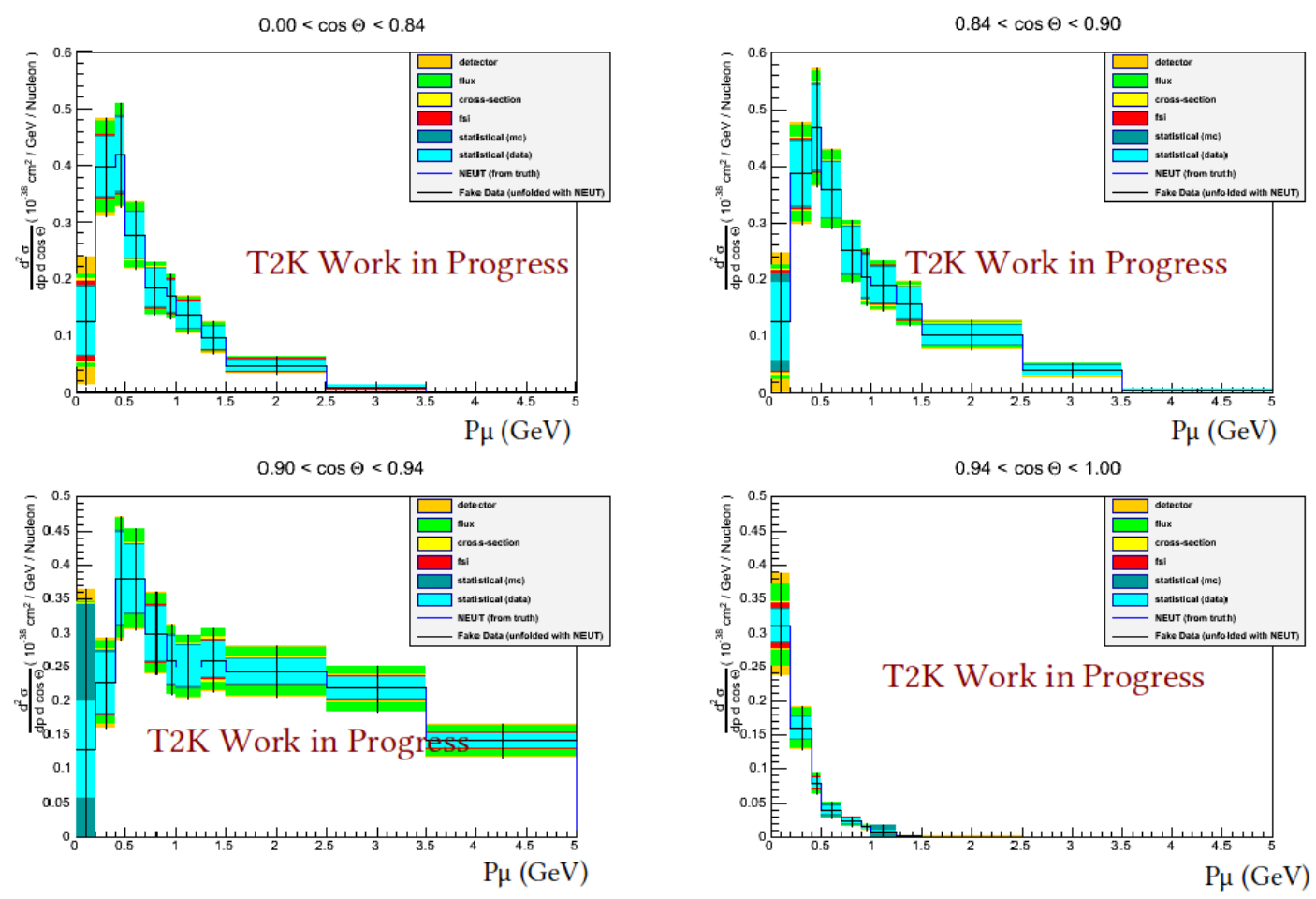

Figure 9: The $\mathrm{T} 2 \mathrm{~K} \mathrm{CC} \pi^{+}$cross section analysis on $\mathrm{CH}$ using NEUT as fake data and unfolding with NEUT.

\section{References}

[1] Y. Fukuda et al. (SK Collaboration),Phys. Rev. Lett. 81, 1562 (1998).

[2] Z. Maki, M. Nakagawa and S. Sakata,Prog. Theor. Phys. 28, 870 (1962).

[3] K. Abe et al. (T2K Collaboration), Nucl. Instrum. Methods A 659, 106 (2011).

[4] K. Abe et al. (T2K Collaboration), Phys. Rev. Lett. 112, 061802 (2014).

[5] J. A. Formaggio and G.P. Zeller Rev. Mod. Phys. 84, 1307 (2012).

[6] P.-A. Amaudruz et al. (T2K ND280 FGD Collaboration), Nucl. Instrum. Methods A 696, 1 (2012).

[7] N. Abgrall et al. (T2K ND280 TPC Collaboration), Nucl. Instrum. Methods A 637, 25 (2011).

[8] S. Assylbekov et al. (T2K ND280 P0D Collaboration), Nucl. Instrum. Methods A 686, 48 (2012).

[9] K. Abe et al. (T2K Collaboration), Phys. Rev. D 87, 092003 (2013).

[10] K. Abe et al. (T2K Collaboration), Phys. Rev. Lett. 113, 241803 (2014).

[11] M. Tzanov (T2K Collaboration), Measurement of the muon neutrino CCQE cross section on carbon with the T2K off-axis near detector in the 9th International Workshop on Neutrino-Nucleus Interactions in the Few-GeV Region (NuINT, UK, 2014).

[12] D. Ruterbories (T2K Collaboration), T2K Neutral Current Elastic Scattering Cross Section in the XVth International Workshop on Neutrino Factories, Super Beams and Beta Beams (NuFact, Beijing, 2013). 